\title{
NEW ESTIMATES FOR THE SOLUTION OF THE LYAPUNOV MATRIX DIFFERENTIAL EQUATION*
}

\author{
JUAN ZHANG ${ }^{\dagger}$ AND JIANZHOU LIU $^{\dagger}$
}

\begin{abstract}
In this paper, by using majorization inequalities, upper bounds on summations of eigenvalues (including the trace) of the solution for the Lyapunov matrix differential equation are obtained. In the limiting cases, the results reduce to bounds of the algebraic Lyapunov matrix equation. The effectiveness of the results are illustrated by numerical examples.
\end{abstract}

Key words. Lyapunov matrix differential equation, Eigenvalue bounds, Majorization inequality.

AMS subject classifications. 93D05, 15A24.

1. Introduction. Consider the Lyapunov matrix differential equation

$$
\dot{P}(t)=A^{T} P(t)+P(t) A+Q, \quad P_{0}=P\left(t_{0}\right),
$$

and the algebraic Lyapunov matrix equation

$$
A^{T} P+P A+Q=0,
$$

where $Q$ is a constant positive semi-definite matrix and $A$ is a constant (Hurwitz) stable real matrix, $P_{0} \geq 0$, and solution of (1.1) and (1.2) are positive semi-definite. The main objective of this paper is to find estimates for the positive semi-definite solution matrices $P(t)$ and $P$ for (1.1) and (1.2), respectively.

The Lyapunov matrix differential equation is important to the stability of linear time-varying systems. In many applications such as signal processing and robust stability analysis, it is important to find reasonable bounds for summations including the trace, and for products including the determinant, of the solution eigenvalues of the Lyapunov matrix differential equation and the algebraic Lyapunov matrix equation (ALE)([1]). Although the exact solution of the Lyapunov equation can be found numerically, the computational burden increases with the dimension of the system matrices. Therefore, it is necessary to find a reasonable estimate for the solution of the Lyapunov equation in stability analysis and control design, such as the upper and lower bounds for the eigenvalues of the solution ([2]).

\footnotetext{
* Received by the editors November 30, 2009. Accepted for publication December 31, 2009. Handling Editor: Daniel Szyld.

${ }^{\dagger}$ Department of Mathematics and Computational Science, Xiangtan University, Xiangtan, Hunan 411105, China (liujz@xtu.edu.cn). Supported in part by Natural Science Foundation of China(10971176).
} 
However, in some cases, we want to know about the mean size of the solution. For example, in the optimal regulator problem, the optimal cost can be written as

$$
J=x_{0}^{T} P x_{0}
$$

where $x_{0} \in R^{n}$ is the initial states of the system and $P$ is the positive definite solution of the algebraic Riccati equation(ARE)

$$
A^{T} P+P A-P R P=-Q .
$$

An interpretation of $\operatorname{tr}(P)$ is that $\operatorname{tr}(P) / n$ is the average value of the cost (1.3) as $x_{0}$ varies over the surface of a unit sphere.

Therefore, considering the applications, many scholars have attempted to pay much attention to the bounds on summations of eigenvalues (including the trace) of the solution for the Lyapunov matrix differential equation, the algebraic Lyapunov matrix equation and the algebraic Riccati equation ([3]-[13]). However, most of the previous bounds are presented under the restrictive assumption that $A+A^{T}$ is negative definite. In this paper, by using majorization inequalities, we will remove this assumption and provide bounds on summations including the trace, of the solution eigenvalues of (1.1) and (1.2).

2. Notations and Previous Results. In the following, let $R^{n \times n}\left(C^{n \times n}\right)$ denote the set of $n \times n$ real (complex) matrices. For $A \in R^{n \times n}$, we assume $A^{T},|A|, A^{-1}$, $\operatorname{tr}(A), \lambda_{i}(A)(1 \leq i \leq n)$ are the transpose, the determinant, the inverse, the trace and $n$ eigenvalues of $A$, respectively. Let $A \in R^{n \times n}$ be an arbitrary symmetric matrix, then we assume that the eigenvalues of $A$ are arranged so that $\lambda_{1}(A) \geq \lambda_{2}(A) \geq \cdots \geq$ $\lambda_{n}(A)$. For any $k=1,2, \ldots, n$, the term $\lambda_{1}(A)+\lambda_{2}(A)+\cdots+\lambda_{k}(A)$ is denoted by $s(A, k)$ and the trace of $A$ is $\operatorname{tr}(A)=s(A, n)$. The notation $A>0(A \geq 0)$ is used to denote that $A$ is a symmetric positive definite (semi-definite) matrix. As in [5], we define a matrix measure $\mu_{2}(A)=\lambda_{1}\left(A_{s}\right)$, where $A_{s}=\frac{1}{2}\left(A+A^{T}\right)$, and suppose there exists some matrix $F>0$ such that $\mu_{F}(A)<0$, where $\mu_{F}(A)=\frac{1}{2} \lambda_{1}\left(F A F^{-1}+A^{T}\right)$. Let $x=\left(x_{1}, x_{2}, \cdots, x_{n}\right)$ be a real $n$-element array which is arranged in non-increasing order. i.e., $x_{[1]} \geq x_{[2]} \geq \cdots \geq x_{[n]}$.

Let $x, y$ be two real $n$-element arrays, if they satisfy

$$
\sum_{i=1}^{k} x_{[i]} \leq \sum_{i=1}^{k} y_{[i]}, \quad k=1,2, \cdots, n,
$$

then $x$ is called weakly majorized by $y$, which is signed by $x \prec_{w} y$.

We give the following lemmas to prove the main results. 
LEMma 2.1. ([14]) Let $H=H^{T} \in R^{n \times n}, U \in C^{k \times n}, 1 \leq k \leq n$, then

$$
\sum_{i=1}^{k} \lambda_{i}(H)=\max _{U U^{T}=I_{k}} \operatorname{tr} U H U^{T}
$$

Lemma 2.2. ([15,p.48]) Let $G \geq 0$ and $H \geq 0$, then for $k=1,2, \cdots, n$,

$$
\sum_{i=1}^{k} \lambda_{i}(G H) \leq \sum_{i=1}^{k} \lambda_{i}(G) \lambda_{i}(H)
$$

with equality when $k=n$.

LEMMA 2.3. ${ }^{([14, p .95, H .3 . b])}$ If $x_{1} \geq \cdots \geq x_{n}, y_{1} \geq \cdots \geq y_{n}$ and $x \prec_{w} y$, then for any real array $u_{1} \geq \cdots \geq u_{n} \geq 0$,

$$
\sum_{i=1}^{k} x_{i} u_{i} \leq \sum_{i=1}^{k} y_{i} u_{i}, \quad k=1,2, \cdots, n .
$$

Lemma 2.4. ([16, p.515]) For any $A_{1}, \cdots, A_{k} \in R^{n \times n}$ and all $m=1,2, \cdots$,

$$
\lim _{m \rightarrow \infty}\left[e^{\frac{A_{1}}{m}} e^{\frac{A_{2}}{m}} \cdots e^{\frac{A_{k}}{m}}\right]^{m}=e^{A_{1}+A_{2}+\cdots+A_{k}} .
$$

Lemma 2.5. For any matrix $A \in R^{n \times n}$, we have

$$
\sum_{i=1}^{k} \lambda_{i}\left(e^{A} e^{A^{T}}\right) \leq \sum_{i=1}^{k} \lambda_{i}\left(e^{A+A^{T}}\right) .
$$

Proof. From [14, ch. 9, A. 1. a], we know $\lambda_{i}(B C)=\lambda_{i}(C B)$, for $B, C \in R^{n \times n}$. Considering Lemma 2.2, for any matrix $X \in R^{n \times n}$ and all $m=1,2, \cdots$, we obtain

$$
\begin{aligned}
\sum_{i=1}^{k} \lambda_{i}\left(X^{m}\left(X^{m}\right)^{T}\right) & =\sum_{i=1}^{k} \lambda_{i}\left(X^{m}\left(X^{T}\right)^{m}\right) \\
& =\sum_{i=1}^{k} \lambda_{i}\left(X\left(X^{m-1}\right)\left(X^{T}\right)^{m-1} X^{T}\right) \\
& =\sum_{i=1}^{k} \lambda_{i}\left(\left(X^{m-1}\right)\left(X^{T}\right)^{m-1} X^{T} X\right)
\end{aligned}
$$




$$
\leq \sum_{i=1}^{k} \lambda_{i}\left(\left(X^{m-1}\right)\left(X^{T}\right)^{m-1}\right) \lambda_{i}\left(X^{T} X\right) .
$$

We proceed by induction on $m$, then in the same manner as (2.7), we have

$$
\sum_{i=1}^{k} \lambda_{i}\left(X^{m}\left(X^{m}\right)^{T}\right) \leq \sum_{i=1}^{k} \lambda_{i}\left(X X^{T}\right)^{m} .
$$

Let $Y=X^{m}$ in (2.8), then we have

$$
\sum_{i=1}^{k} \lambda_{i}\left(Y Y^{T}\right) \leq \sum_{i=1}^{k} \lambda_{i}\left[Y^{\frac{1}{m}}\left(Y^{T}\right)^{\frac{1}{m}}\right]^{m}
$$

Then for any matrix $A \in R^{n \times n}$, if we choose $e^{A}=Y$ in (2.9), we obtain

$$
\sum_{i=1}^{k} \lambda_{i}\left(e^{A} e^{A^{T}}\right) \leq \sum_{i=1}^{k} \lambda_{i}\left[e^{\frac{A}{m}} e^{\frac{A^{T}}{m}}\right]^{m} .
$$

From Lemma 2.4, let $m \rightarrow \infty$ in (2.10), then we have

$$
\begin{aligned}
\sum_{i=1}^{k} \lambda_{i}\left(e^{A} e^{A^{T}}\right) & \leq \lim _{m \rightarrow \infty} \sum_{i=1}^{k} \lambda_{i}\left[e^{\frac{A}{m}} e^{\frac{A^{T}}{m}}\right]^{m} \\
& =\sum_{i=1}^{k} \lambda_{i}\left[\lim _{m \rightarrow \infty}\left(e^{\frac{A}{m}} e^{\frac{A^{T}}{m}}\right)^{m}\right] \\
& =\sum_{i=1}^{k} \lambda_{i}\left(e^{A+A^{T}}\right) .
\end{aligned}
$$

This completes the proof.

3. Main Results. In this section, we first give a new upper bound on summations including the trace, of the solution eigenvalues of (1.1) under the single assumption that $A$ is a constant (Hurwitz) stable matrix. Then, we give a modification of (1.1), and give a new upper bound on summations including the trace, of the solution eigenvalues of (1.1).

THEOREM 3.1. Suppose that the real matrix $A$ is stable and $A+A^{T}$ is nonsingular, then we have

$$
s(P(t), k) \leq \sum_{i=1}^{k} \lambda_{i}\left(P\left(t_{0}\right)\right) e^{\lambda_{i}\left(A+A^{T}\right)\left(t-t_{0}\right)}-\sum_{i=1}^{k} \frac{\lambda_{i}(Q)}{\lambda_{i}\left(A+A^{T}\right)}
$$




$$
+\sum_{i=1}^{k} \frac{\lambda_{i}(Q) e^{\lambda_{i}\left(A+A^{T}\right)\left(t-t_{0}\right)}}{\lambda_{i}\left(A+A^{T}\right)}
$$

Proof. The solution of (1.1) can be expressed as

$$
P(t)=e^{A^{T}\left(t-t_{0}\right)} P\left(t_{0}\right) e^{A\left(t-t_{0}\right)}+\int_{t_{0}}^{t} e^{A^{T}(t-s)} Q e^{A(t-s)} d s .
$$

Since $P(t)=P^{T}(t)$, then from Lemma 2.1, we know there exists a $k \times n$ functional matrix $U(t)$ such that

$$
\begin{aligned}
s(P(t), k)= & \sum_{i=1}^{k} \lambda_{i}(P(t)) \\
= & \max _{U(t) U^{T}(t)=I_{k}} \operatorname{tr}\left(U(t) P(t) U^{T}(t)\right) \\
= & \max _{U(t) U^{T}(t)=I_{k}}\left[\operatorname{tr}\left(U(t) e^{A^{T}\left(t-t_{0}\right)} P\left(t_{0}\right) e^{A\left(t-t_{0}\right)} U^{T}(t)\right)\right. \\
& \left.+\operatorname{tr}\left(U(t) \int_{t_{0}}^{t} e^{A^{T}(t-s)} Q e^{A(t-s)} d s U^{T}(t)\right)\right] \\
\leq & \max _{U(t) U^{T}(t)=I_{k}} \operatorname{tr}\left(U(t) e^{A^{T}\left(t-t_{0}\right)} P\left(t_{0}\right) e^{A\left(t-t_{0}\right)} U^{T}(t)\right) \\
& \quad \max _{U(t) U^{T}(t)=I_{k}} \operatorname{tr}\left(U(t) \int_{t_{0}}^{t} e^{A^{T}(t-s)} Q e^{A(t-s)} d s U^{T}(t)\right) .
\end{aligned}
$$

Note that there is no relation between the functional matrix $U(t)$ and the integral variable $s$, and for any function matrix $w(t)$ with order $n$, we have

$$
\operatorname{tr} \int_{t_{0}}^{t} w(s) d s=\sum_{i=1}^{n} \int_{t_{0}}^{t} w_{i i}(s) d s=\int_{t_{0}}^{t} \sum_{i=1}^{n} w_{i i}(s) d s=\int_{t_{0}}^{t} \operatorname{tr} w(s) d s .
$$

Since $P\left(t_{0}\right), Q \geq 0$, then from Lemma 2.2, we obtain

$$
\begin{aligned}
s(P(t), k) \leq & \max _{U(t) U^{T}(t)=I_{k}} \operatorname{tr}\left(P\left(t_{0}\right) e^{A\left(t-t_{0}\right)} U^{T}(t) U(t) e^{A^{T}\left(t-t_{0}\right)}\right) \\
& +\max _{U(t) U^{T}(t)=I_{k}} \operatorname{tr} \int_{t_{0}}^{t} U(t) e^{A^{T}(t-s)} Q e^{A(t-s)} U^{T}(t) d s
\end{aligned}
$$




$$
\begin{aligned}
& =\max _{U(t) U^{T}(t)=I_{k}} \operatorname{tr}\left(P\left(t_{0}\right) e^{A\left(t-t_{0}\right)} U^{T}(t) U(t) e^{A^{T}\left(t-t_{0}\right)}\right) \\
& +\max _{U(t) U^{T}(t)=I_{k}} \int_{t_{0}}^{t} \operatorname{tr}\left(U(t) e^{A^{T}(t-s)} Q e^{A(t-s)} U^{T}(t)\right) d s \\
& =\max _{U(t) U^{T}(t)=I_{k}} \sum_{i=1}^{k} \lambda_{i}\left(P\left(t_{0}\right) e^{A\left(t-t_{0}\right)} U^{T}(t) U(t) e^{A^{T}\left(t-t_{0}\right)}\right) \\
& +\max _{U(t) U^{T}(t)=I_{k}} \int_{t_{0}}^{t} \sum_{i=1}^{k} \lambda_{i}\left(U(t) e^{A^{T}(t-s)} Q e^{A(t-s)} U^{T}(t)\right) d s \\
& =\max _{U(t) U^{T}(t)=I_{k}} \sum_{i=1}^{k} \lambda_{i}\left(P\left(t_{0}\right) e^{A\left(t-t_{0}\right)} U^{T}(t) U(t) e^{A^{T}\left(t-t_{0}\right)}\right) \\
& +\max _{U(t) U^{T}(t)=I_{k}} \int_{t_{0}}^{t} \sum_{i=1}^{k} \lambda_{i}\left(Q e^{A(t-s)} U^{T}(t) U(t) e^{A^{T}(t-s)}\right) d s \\
& \leq \max _{U(t) U^{T}(t)=I_{k}} \sum_{i=1}^{k} \lambda_{i}\left(P\left(t_{0}\right)\right) \lambda_{i}\left(e^{A\left(t-t_{0}\right)} U^{T}(t) U(t) e^{A^{T}\left(t-t_{0}\right)}\right) \\
& +\max _{U(t) U^{T}(t)=I_{k}} \int_{t_{0}}^{t} \sum_{i=1}^{k} \lambda_{i}(Q) \lambda_{i}\left(e^{A(t-s)} U^{T}(t) U(t) e^{A^{T}(t-s)}\right) d s \\
& =\max _{U(t) U^{T}(t)=I_{k}} \sum_{i=1}^{k} \lambda_{i}\left(P\left(t_{0}\right)\right) \lambda_{i}\left(e^{A^{T}\left(t-t_{0}\right)} e^{A\left(t-t_{0}\right)} U^{T}(t) U(t)\right) \\
& +\max _{U(t) U^{T}(t)=I_{k}} \int_{t_{0}}^{t} \sum_{i=1}^{k} \lambda_{i}(Q) \lambda_{i}\left(e^{A^{T}(t-s)} e^{A(t-s)} U^{T}(t) U(t)\right) d s .
\end{aligned}
$$

For any $l=1,2, \cdots, k$, put $u_{i}=\lambda_{i}\left(P\left(t_{0}\right)\right) \geq 0$ and $u_{i}=\lambda_{i}(Q) \geq 0$ in $(3.2)$, from Lemma 2.2 and Lemma 2.3, we have

$$
\sum_{i=1}^{k} \lambda_{i}\left(P\left(t_{0}\right)\right) \lambda_{i}\left(e^{A^{T}\left(t-t_{0}\right)} e^{A\left(t-t_{0}\right)} U^{T}(t) U(t)\right)
$$




$$
\begin{gathered}
\leq \sum_{i=1}^{k} \lambda_{i}\left(P\left(t_{0}\right)\right) \lambda_{i}\left(e^{A^{T}\left(t-t_{0}\right)} e^{A\left(t-t_{0}\right)}\right) \lambda_{i}\left(U^{T}(t) U(t)\right), \\
\sum_{i=1}^{k} \lambda_{i}(Q) \lambda_{i}\left(e^{A^{T}(t-s)} e^{A(t-s)} U^{T}(t) U(t)\right) \\
\leq \sum_{i=1}^{k} \lambda_{i}(Q) \lambda_{i}\left(e^{A^{T}(t-s)} e^{A(t-s)}\right) \lambda_{i}\left(U^{T}(t) U(t)\right) .
\end{gathered}
$$

Therefore, by Lemma 2.5 and (3.2), (3.3), (3.4), we obtain

$$
\begin{aligned}
& s(P(t), k) \leq \max _{U(t) U^{T}(t)=I_{k}} \sum_{i=1}^{k} \lambda_{i}\left(P\left(t_{0}\right)\right) \lambda_{i}\left(e^{A^{T}\left(t-t_{0}\right)} e^{A\left(t-t_{0}\right)}\right) \lambda_{i}\left(U^{T}(t) U(t)\right) \\
& +\max _{U(t) U^{T}(t)=I_{k}} \int_{t_{0}}^{t} \sum_{i=1}^{k} \lambda_{i}(Q) \lambda_{i}\left(e^{A^{T}(t-s)} e^{A(t-s)}\right) \lambda_{i}\left(U^{T}(t) U(t)\right) d s \\
& \leq \max _{U(t) U^{T}(t)=I_{k}} \sum_{i=1}^{k} \lambda_{i}\left(P\left(t_{0}\right)\right) \lambda_{i}\left(e^{\left(A^{T}+A\right)\left(t-t_{0}\right)}\right) \lambda_{i}\left(U(t) U^{T}(t)\right) \\
& +\max _{U(t) U^{T}(t)=I_{k}} \int_{t_{0}}^{t} \sum_{i=1}^{k} \lambda_{i}(Q) \lambda_{i}\left(e^{\left(A^{T}+A\right)(t-s)}\right) \lambda_{i}\left(U(t) U^{T}(t)\right) d s \text { by }(2.6) \\
& =\sum_{i=1}^{k} \lambda_{i}\left(P\left(t_{0}\right)\right) e^{\lambda_{i}\left(A^{T}+A\right)\left(t-t_{0}\right)}+\int_{t_{0}}^{t} \sum_{i=1}^{k} \lambda_{i}(Q) e^{\lambda_{i}\left(A^{T}+A\right)(t-s)} d s \\
& =\sum_{i=1}^{k} \lambda_{i}\left(P\left(t_{0}\right)\right) e^{\lambda_{i}\left(A^{T}+A\right)\left(t-t_{0}\right)}+\left.\sum_{i=1}^{k}\left(-\frac{\lambda_{i}(Q) e^{\lambda_{i}\left(A+A^{T}\right)(t-s)}}{\lambda_{i}\left(A+A^{T}\right)}\right)\right|_{t_{0}} ^{t} \\
& =\sum_{i=1}^{k} \lambda_{i}\left(P\left(t_{0}\right)\right) e^{\lambda_{i}\left(A^{T}+A\right)\left(t-t_{0}\right)}-\sum_{i=1}^{k} \frac{\lambda_{i}(Q)}{\lambda_{i}\left(A+A^{T}\right)} \\
& +\sum_{i=1}^{k} \frac{\lambda_{i}(Q) e^{\lambda_{i}\left(A+A^{T}\right)\left(t-t_{0}\right)}}{\lambda_{i}\left(A+A^{T}\right)}
\end{aligned}
$$


This completes the proof. $\square$

Let $k=n$, from (3.1), we obtain the following Corollary about (1.1).

Corollary 3.2. Suppose that the real matrix $A$ is stable and $A+A^{T}$ is nonsingular, then we have

$\operatorname{tr}(P(t)) \leq \sum_{i=1}^{n} \lambda_{i}\left(P\left(t_{0}\right)\right) e^{\lambda_{i}\left(A+A^{T}\right)\left(t-t_{0}\right)}-\sum_{i=1}^{n} \frac{\lambda_{i}(Q)}{\lambda_{i}\left(A+A^{T}\right)}+\sum_{i=1}^{n} \frac{\lambda_{i}(Q) e^{\lambda_{i}\left(A+A^{T}\right)\left(t-t_{0}\right)}}{\lambda_{i}\left(A+A^{T}\right)}$.

REMARK 3.3. Note that for $i=1, \cdots, n$, we have $\lambda_{i}\left(P\left(t_{0}\right)\right) \leq \lambda_{1}\left(P\left(t_{0}\right)\right), \lambda_{i}(Q) \leq$ $\lambda_{1}(Q)$. Then

$$
\begin{aligned}
& \sum_{i=1}^{n} \lambda_{i}\left(P\left(t_{0}\right)\right) e^{\lambda_{i}\left(A+A^{T}\right)\left(t-t_{0}\right)}-\sum_{i=1}^{n} \frac{\lambda_{i}(Q)}{\lambda_{i}\left(A+A^{T}\right)}+\sum_{i=1}^{n} \frac{\lambda_{i}(Q) e^{\lambda_{i}\left(A+A^{T}\right)\left(t-t_{0}\right)}}{\lambda_{i}\left(A+A^{T}\right)} \\
& \leq \lambda_{1}\left(P\left(t_{0}\right)\right) \operatorname{tr}\left(e^{\left(A+A^{T}\right)\left(t-t_{0}\right)}\right)-\lambda_{1}(Q) \operatorname{tr}\left(\left(A+A^{T}\right)^{-1}\right)+\lambda_{1}(Q) \sum_{i=1}^{n} \frac{e^{\lambda_{i}\left(A+A^{T}\right)\left(t-t_{0}\right)}}{\lambda_{i}\left(A+A^{T}\right)} .
\end{aligned}
$$

This implies that (3.1) is better than Theorem 3.1 in [5].

If $\lambda_{1}\left(A+A^{T}\right)<0$, when $t \rightarrow \infty$ in (3.1) and (3.6), we obtain estimates for the algebraic Lyapunov matrix equation (1.2) and we have the following corollaries.

Corollary 3.4. Suppose that the real matrix $A$ is stable and $\lambda_{1}\left(A+A^{T}\right)<0$, then we have

$$
s(P, k) \leq-\sum_{i=1}^{k} \frac{\lambda_{i}(Q)}{\lambda_{i}\left(A+A^{T}\right)} .
$$

Corollary 3.5. Suppose that the real matrix $A$ is stable and $\lambda_{1}\left(A+A^{T}\right)<0$, then we have

$$
\operatorname{tr}(P) \leq-\sum_{i=1}^{n} \frac{\lambda_{i}(Q)}{\lambda_{i}\left(A+A^{T}\right)}
$$

Note that (3.8) is Theorem 3.5 in [5].

To remove the restrictive assumption that $\mu_{2}(A)<0$, we give a modification of (1.1) as follows:

$$
\dot{\widetilde{P}}(t)=\widetilde{A}^{T} \widetilde{P}(t)+\widetilde{P}(t) \widetilde{A}+\widetilde{Q}
$$




$$
\widetilde{A}=T A T^{-1}, \widetilde{P}(t)=T^{-1} P(t) T^{-1}, \widetilde{Q}=T^{-1} Q T^{-1},
$$

with $T=\sqrt{F}, F>0$ such that $\mu_{F}(A)<0$. Obviously, $\dot{\widetilde{P}}(t)=T^{-1} \dot{P}(t) T^{-1}$.

Applying Lemma 2.3 and Theorem 3.1 to (3.9), we can easily obtain the following theorem.

THEOREM 3.6. Let $F$ be a positive definite matrix satisfying $\mu_{F}(A)<0$, then

$$
\begin{aligned}
s(P(t), k) \leq & \sum_{i=1}^{k} \lambda_{i}(F) \lambda_{i}\left(\widetilde{P}\left(t_{0}\right)\right)\left(e^{\lambda_{i}\left(\widetilde{A}+\widetilde{A}^{T}\right)\left(t-t_{0}\right)}\right)-\sum_{i=1}^{k} \frac{\lambda_{i}(F) \lambda_{i}\left(F^{-1} Q\right)}{\lambda_{i}\left(F A F^{-1}+A^{T}\right)} \\
& +\sum_{i=1}^{k} \frac{\lambda_{i}(F) \lambda_{i}\left(F^{-1} Q\right) e^{\lambda_{i}\left(F A F^{-1}+A^{T}\right)\left(t-t_{0}\right)}}{\lambda_{i}\left(F A F^{-1}+A^{T}\right)}
\end{aligned}
$$

Proof. Note that

$$
\begin{gathered}
T=\sqrt{F}, \quad \lambda_{i}(\widetilde{Q})=\lambda_{i}\left(T^{-1} Q T^{-1}\right)=\lambda_{i}\left(F^{-1} Q\right), \\
\lambda_{i}\left(\widetilde{A}+\widetilde{A}^{T}\right)=\lambda_{i}\left(T A T^{-1}+T^{-1} A^{T} T\right)=\lambda_{i}\left(F A F^{-1}+A^{T}\right) .
\end{gathered}
$$

So $\mu_{F}(A)<0$ is equivalent to $\mu_{2}(\widetilde{A})<0$, i.e., $\widetilde{A}+\widetilde{A}^{T}$ is nonsingular. Then from Theorem 3.1, we obtain

$$
\begin{aligned}
& \text { 11) } s(\widetilde{P}(t), k)=\sum_{i=1}^{k} \lambda_{i}(\widetilde{P}(t)) \\
& \leq \sum_{i=1}^{k} \lambda_{i}\left(\widetilde{P}\left(t_{0}\right)\right) e^{\lambda_{i}\left(\widetilde{A}+\widetilde{A}^{T}\right)\left(t-t_{0}\right)}-\sum_{i=1}^{k} \frac{\lambda_{i}(\widetilde{Q})}{\lambda_{i}\left(\widetilde{A}+\widetilde{A}^{T}\right)}+\sum_{i=1}^{k} \frac{\lambda_{i}(\widetilde{Q}) e^{\lambda_{i}\left(\widetilde{A}+\widetilde{A}^{T}\right)\left(t-t_{0}\right)}}{\lambda_{i}\left(\widetilde{A}+\widetilde{A}^{T}\right)} .
\end{aligned}
$$

And (3.11) can be written as

$$
\begin{aligned}
& \sum_{i=1}^{k}\left[\lambda_{i}(\widetilde{P}(t))-\frac{\lambda_{i}\left(F^{-1} Q\right)}{\lambda_{i}\left(F A F^{-1}+A^{T}\right)} e^{\lambda_{i}\left(F A F^{-1}+A^{T}\right)\left(t-t_{0}\right)}\right] \\
& \leq \sum_{i=1}^{k}\left[\lambda_{i}\left(\widetilde{P}\left(t_{0}\right)\right) e^{\lambda_{i}\left(\widetilde{A}+\widetilde{A}^{T}\right)\left(t-t_{0}\right)}-\frac{\lambda_{i}\left(F^{-1} Q\right)}{\lambda_{i}\left(F A F^{-1}+A^{T}\right)}\right]
\end{aligned}
$$

As

$$
\lambda_{1}(\widetilde{P}(t)) \geq \cdots \geq \lambda_{n}(\widetilde{P}(t)) \geq 0
$$




$$
\begin{gathered}
\lambda_{1}\left(\widetilde{P}\left(t_{0}\right)\right) \geq \cdots \geq \lambda_{n}\left(\widetilde{P}\left(t_{0}\right)\right) \geq 0, \\
\lambda_{1}\left(F^{-1} Q\right) \geq \cdots \geq \lambda_{n}\left(F^{-1} Q\right) \geq 0, \\
e^{\lambda_{1}\left(F A F^{-1}+A^{T}\right)\left(t-t_{0}\right)} \geq \cdots \geq e^{\lambda_{n}\left(F A F^{-1}+A^{T}\right)\left(t-t_{0}\right)} \geq 0, \\
{\left[-\lambda_{1}\left(F A F^{-1}+A^{T}\right)\right]^{-1}} \\
\geq \cdots \geq\left[-\lambda_{n}\left(F A F^{-1}+A^{T}\right)\right]^{-1} \geq 0 .
\end{gathered}
$$

Then

$$
\begin{gathered}
\lambda_{1}(\widetilde{P}(t))-\frac{\lambda_{1}\left(F^{-1} Q\right)}{\lambda_{1}\left(F A F^{-1}+A^{T}\right)} e^{\lambda_{1}\left(F A F^{-1}+A^{T}\right)\left(t-t_{0}\right)} \geq \\
\cdots \geq \lambda_{n}(\widetilde{P}(t))-\frac{\lambda_{n}\left(F^{-1} Q\right)}{\lambda_{n}\left(F A F^{-1}+A^{T}\right)} e^{\lambda_{n}\left(F A F^{-1}+A^{T}\right)\left(t-t_{0}\right)} \geq 0, \\
\lambda_{1}\left(\widetilde{P}\left(t_{0}\right)\right) e^{\lambda_{1}\left(\widetilde{A}+\widetilde{A}^{T}\right)\left(t-t_{0}\right)}-\frac{\lambda_{1}\left(F^{-1} Q\right)}{\lambda_{1}\left(F A F^{-1}+A^{T}\right)} \geq \\
\cdots \geq \lambda_{n}\left(\widetilde{P}\left(t_{0}\right)\right) e^{\lambda_{n}\left(\widetilde{A}+\widetilde{A}^{T}\right)\left(t-t_{0}\right)}-\frac{\lambda_{n}\left(F^{-1} Q\right)}{\lambda_{n}\left(F A F^{-1}+A^{T}\right)} \geq 0 .
\end{gathered}
$$

Let

$$
\begin{gathered}
x_{i}=\lambda_{i}(\widetilde{P}(t))-\frac{\lambda_{i}\left(F^{-1} Q\right)}{\lambda_{i}\left(F A F^{-1}+A^{T}\right)} e^{\lambda_{i}\left(F A F^{-1}+A^{T}\right)\left(t-t_{0}\right)}, \\
y_{i}=\lambda_{i}\left(\widetilde{P}\left(t_{0}\right)\right) e^{\lambda_{i}\left(\widetilde{A}+\widetilde{A}^{T}\right)\left(t-t_{0}\right)}-\frac{\lambda_{i}\left(F^{-1} Q\right)}{\lambda_{i}\left(F A F^{-1}+A^{T}\right)} .
\end{gathered}
$$

From (3.12), then we have

$$
\left(x_{1}, x_{2}, \cdots, x_{n}\right) \prec_{w}\left(y_{1}, y_{2}, \cdots, y_{n}\right) .
$$

Choose $u_{i}=\lambda_{i}(F)$, since $\lambda_{1}(F) \geq \lambda_{2}(F) \geq \cdots \geq \lambda_{n}(F) \geq 0$. Then applying Lemma 2.3 to (3.12), we have

$$
\begin{aligned}
& \sum_{i=1}^{k}\left[\lambda_{i}(\widetilde{P}(t))-\frac{\lambda_{i}\left(F^{-1} Q\right)}{\lambda_{i}\left(F A F^{-1}+A^{T}\right)} e^{\lambda_{i}\left(F A F^{-1}+A^{T}\right)\left(t-t_{0}\right)}\right] \lambda_{i}(F) \\
& \leq \sum_{i=1}^{k}\left[\lambda_{i}\left(\widetilde{P}\left(t_{0}\right)\right) e^{\lambda_{i}\left(\widetilde{A}+\widetilde{A}^{T}\right)\left(t-t_{0}\right)}-\frac{\lambda_{i}\left(F^{-1} Q\right)}{\lambda_{i}\left(F A F^{-1}+A^{T}\right)}\right] \lambda_{i}(F) .
\end{aligned}
$$


From (3.13), using the relationship $\sum_{i=1}^{k} \lambda_{i}(P(t))=\sum_{i=1}^{k} \lambda_{i}(\widetilde{P}(t) F)$ and Lemma 2.2, it follows that

$$
\begin{aligned}
\sum_{i=1}^{k} \lambda_{i}(P(t)) & =\sum_{i=1}^{k} \lambda_{i}(\widetilde{P} F) \\
& \leq \sum_{i=1}^{k} \lambda_{i}(\widetilde{P}(t)) \lambda_{i}(F) \\
& \leq \sum_{i=1}^{k}\left[\lambda_{i}\left(\widetilde{P}\left(t_{0}\right)\right) e^{\lambda_{i}\left(\widetilde{A}+\widetilde{A}^{T}\right)\left(t-t_{0}\right)}-\frac{\lambda_{i}\left(F^{-1} Q\right)}{\lambda_{i}\left(F A F^{-1}+A^{T}\right)}\right] \lambda_{i}(F) \\
& +\sum_{i=1}^{k} \frac{\lambda_{i}(F) \lambda_{i}\left(F^{-1} Q\right) e^{\lambda_{i}\left(F A F^{-1}+A^{T}\right)\left(t-t_{0}\right)}}{\lambda_{i}\left(F A F^{-1}+A^{T}\right)} .
\end{aligned}
$$

This completes the proof. $\square$

Let $k=n$, from (3.10), we obtain the following corollary about (1.1).

Corollary 3.7. Let $F$ be a positive definite matrix satisfying $\mu_{F}(A)<0$, then

$$
\begin{aligned}
& \operatorname{tr}(P(t)) \leq \sum_{i=1}^{n} \lambda_{i}(F) \lambda_{i}\left(\widetilde{P}\left(t_{0}\right)\right) e^{\lambda_{i}\left(\widetilde{A}+\widetilde{A}^{T}\right)\left(t-t_{0}\right)} \\
& -\sum_{i=1}^{n} \frac{\lambda_{i}(F) \lambda_{i}\left(F^{-1} Q\right)}{\lambda_{i}\left(F A F^{-1}+A^{T}\right)}+\sum_{i=1}^{n} \frac{\lambda_{i}(F) \lambda_{i}\left(F^{-1} Q\right) e^{\lambda_{i}\left(F A F^{-1}+A^{T}\right)\left(t-t_{0}\right)}}{\lambda_{i}\left(F A F^{-1}+A^{T}\right)} .
\end{aligned}
$$

When $t \rightarrow \infty$ in (3.10) and (3.14), we obtain estimates for the algebraic Lyapunov matrix equation (1.2) and we have the following corollaries.

Corollary 3.8. Let $F$ be a positive definite matrix satisfying $\mu_{F}(A)<0$, then

$$
s(P, k) \leq-\sum_{i=1}^{k} \frac{\lambda_{i}(F) \lambda_{i}\left(F^{-1} Q\right)}{\lambda_{i}\left(F A F^{-1}+A^{T}\right)} .
$$

Corollary 3.9. Let $F$ be a positive definite matrix satisfying $\mu_{F}(A)<0$, then

$$
\operatorname{tr}(P) \leq-\sum_{i=1}^{n} \frac{\lambda_{i}(F) \lambda_{i}\left(F^{-1} Q\right)}{\lambda_{i}\left(F A F^{-1}+A^{T}\right)}
$$


REMARK 3.10. Note that for $i=1, \cdots, n$, we have $\lambda_{i}(F) \leq \lambda_{1}(F)$. Then

$$
-\sum_{i=1}^{n} \frac{\lambda_{i}(F) \lambda_{i}\left(F^{-1} Q\right)}{\lambda_{i}\left(F A F^{-1}+A^{T}\right)} \leq-\lambda_{1}(F) \sum_{i=1}^{n} \frac{\lambda_{i}\left(F^{-1} Q\right)}{\lambda_{i}\left(F A F^{-1}+A^{T}\right)} .
$$

This implies that (3.15) is better than Theorem 3.7 in [5].

4. Numerical Examples. In this section, we present examples to illustrate the effectiveness of the main results.

EXAMPLE 4.1. Let

$$
\begin{gathered}
A=\left(\begin{array}{ccc}
-1 & -2 & 0 \\
1 & -1 & 5 \\
0 & -4 & -1
\end{array}\right), \quad Q=\left(\begin{array}{lll}
1 & 0 & 0 \\
0 & 1 & 0 \\
0 & 0 & 1
\end{array}\right), \\
P\left(t_{0}\right)=P(0)=\left(\begin{array}{ccc}
3 & 1 & -2 \\
1 & 4 & 0 \\
-2 & 0 & 2
\end{array}\right) .
\end{gathered}
$$

Case 1. $k=2, t=0.5$.

By (3.1), we obtain

$$
s(P(0.5)), 2) \leq 4.0643 .
$$

However, ([5, Theorem 3.1]) can't be used.

Case 2. $k=3, t=0.5$.

By ([5, Theorem 3.1]), we obtain

$$
\operatorname{tr}(P(0.5)) \leq 6.6676 .
$$

By (3.1), we obtain

$$
s(P(0.5)), 3)=\operatorname{tr}(P(0.5)) \leq 6.1845 .
$$

Thus, (3.1) is better than ([5, Theorem 3.1]).

EXAMPLE 4.2. Let

$$
A=\left(\begin{array}{ccc}
-1 & 2 & 0 \\
0 & -1 & 0 \\
0 & 0 & -1
\end{array}\right), \quad Q=\left(\begin{array}{lll}
1 & 0 & 0 \\
0 & 1 & 0 \\
0 & 0 & 1
\end{array}\right)
$$


Since $\mu_{2}(A)=0$, we can not use Theorem 3.1.

We choose $F=\left(\begin{array}{ccc}\varepsilon^{2} & 0 & 0 \\ 0 & 1 & 0 \\ 0 & 0 & 1\end{array}\right)$, where $\varepsilon>0$ is to be determined, we choose $\varepsilon=0.5$.

Using Corollary 3.2 in [5], we have

$$
\operatorname{tr}(P) \leq 6.0000 .
$$

Using Theorem 3.6 in [5], we have

$$
\operatorname{tr}(P) \leq 5.0000 .
$$

Using Theorem 3.7 in [5], we have

$$
\operatorname{tr}(P) \leq 4.8333 .
$$

Using (3.15), we have

$$
\operatorname{tr}(P) \leq 4.5833
$$

From this example, (3.15) is better than some results in [5].

Acknowledgment. The authors would like to thank Professor Daniel Szyld and the referees for the very helpful comments and suggestions to improve the contents and presentation of this paper.

\section{REFERENCES}

[1] S. Barnett and C. Storey. Matrix Methods in Stability Theory. New York: Barnes and Noble, 1970.

[2] T. Mori, E. Noldus, and M. Kuwahara A way to stabilize linear systems with delayed state. Automatica, 19:571-574, 1983.

[3] T. Mori, N. Fukuma, and M. Kuwahara. On the Lyapunov matrix differential equation. IEEE Trans. Automat. Contr., 31:868-869, 1986.

[4] T. Mori, N. Fukuma, and M. Kuwahara. Bounds in the Lyapunov matrix differential equation. IEEE Trans. Automat. Contr., 32:55-57, 1987.

[5] Y. Fang, K. A. Loparo and X. Feng. New estimates for solution of Lyapunov equation. IEEE Trans. Automat. Contr., 42:408-411, 1997.

[6] S. D. Wang, T. S. Kuo, and C. F. Hsu. The bounds on the solution of the algebraic Riccati and Lyapunov equation. IEEE Trans. Automat. Contr., 31:654-656, 1986. 
[7] J. M. Saniuk and I. B. Rhodes. A matrix inequality associated with bounds on solutions of algebraic Riccati and Lyapunov equations. IEEE Trans. Automat. Contr., 32:739-740, 1987.

[8] T. Mori. Comments on A matrix inequality associated with bounds on solutions of algebraic Riccati and Lyapunov equations. IEEE Trans. Automat. Contr., 33:1088-1091, 1988.

[9] N. Komaroff. Upper summation and product bounds for solution eigenvalues of the Lyapunov matrix equation. IEEE Trans. Automat. Contr., 37:1040-1042, 1992.

[10] W. H. Kwon, Y. S. Moon, and S. C. Ahn. Bounds on solutions of algebraic Riccati and Lyapunov equations: A survey and new results. Int. J. Control, 64:377-389, 1996.

[11] S. Savov and I. Popchev. New upper estimates for the solution of the continuous algebraic Lyapunov equation. IEEE Trans. Automat. Contr., 49:1841-1842, 2004.

[12] W. Xing, Q. Zhang and Q. Wang. A trace bound for a general square matrix product. IEEE Trans. Automat. Contr., 45:1563-1565, 2000.

[13] Jianzhou Liu and LingLi He. A new trace bound for a general square matrix product. IEEE Trans. Automat. Contr., 52(2):349-352, 2007.

[14] A. W. Marshall and I. Olkin. Inequalities Theory of Majorsation and Its Applications. Academic Press, New York, 1979.

[15] Fuzhen Zhang. The Schur complement and its applications. Springer-Verlag, New York, 2005.

[16] Roger A. Horn, Charles R. Johnson. Topics in Matrix Analysis. Cambridge University Press, 1991. 